\title{
TNO surface ices
}

\section{Observations of the TNO $55638\left(2002 \mathrm{VE}_{95}\right)$ and analysis of the population's spectral properties ${ }^{\star}$}

\author{
M. A. Barucci ${ }^{1}$, F. Merlin ${ }^{1}$, E. Dotto ${ }^{2}$, A. Doressoundiram ${ }^{1}$, and C. de Bergh ${ }^{1}$ \\ 1 LESIA, Observatoire de Paris, 92195 Meudon Principal Cedex, France \\ e-mail: antonella.barucci@obspm.fr \\ 2 INAF, Observatorio Astronomico di Roma, via Frascati 33, 00040 Monteporzio Catone (Roma), Italy
}

Received 1 February 2006 / Accepted 25 April 2006

ABSTRACT

\begin{abstract}
Aims. We investigate the surface composition of Centaurs and Trans-Neptunian objects (TNOs) to get constraints on the formation and the evolution of this population.

Methods. We report visible and near-infrared spectroscopic observations of the Plutino 55638 (2002 VE 5 ) obtained at VLT-ESO. The surface model has been computed using two types of radiative transfer models considering geographical and intimate mixtures of different materials. The obtained results have been compared with those for objects having near-infrared spectra available in the literature. The whole sample of 32 objects has been analyzed, in particular the presence of ices has been investigated in relation to their surface characteristics (taxonomic groups), their dynamical properties and the object sizes.

Results. The main result is the clear detection of $\mathrm{H}_{2} \mathrm{O}$ and $\mathrm{CH}_{3} \mathrm{OH}$ (or a similar molecule) on the surface of 55638. The analysis of the whole sample shows that there are no obvious trends, but the larger objects seem to be icier. The BB group also seems to show more ice content on the surface, whereas RR group may contain more organic material.
\end{abstract}

Key words. radiative transfer - techniques: spectroscopic - Kuiper Belt

\section{Introduction}

The Trans-Neptunian region is populated by icy bodies believed to retain the most pristine and least altered material of the Solar System. Since 1992, when the detection of the first Trans-Neptunian object (TNO) was reported by Jewitt \& Luu (1993), more than 1000 such objects have been found with different orbits classified in several dynamical classes. Classical objects have orbits with low eccentricities and semi-major axes between about 42 and $48 \mathrm{AU}$; Resonant objects are trapped in resonances with Neptune, the majority of them are located in or near the 3:2 mean motion resonance; Scattered objects have high-eccentricity, high-inclination orbits and a perihelion distance near $q=35 \mathrm{AU}$; while the Extended scattered disk objects are located at distances too large for gravitational encounters with Neptune. The Centaurs with unstable orbits between those of Jupiter and Neptune can also be associated with the TNO population. Centaurs seem to come from TNOs and are injected into their present orbit by gravitational instabilities and collisions (Duncan et al. 1995).

The investigation of the surface composition of these icy bodies provide essential information about the conditions in the early Solar System environment at large distances from the Sun. Studies of the physical properties of these objects are still limited by their faintness, and particularly lacking is the information on the compositional properties of their surfaces.

* Based on observations obtained at the VLT Observatory, Cerro Paranal of European Southern Observatory, ESO, Chile, in the framework of programs 072.C-0063 and 074.C-0121.
Many photometric surveys have been performed, providing data for a large number of objects, and relevant statistical analyses have been applied (see Barucci \& Peixinho 2006, for a review). The large variety of colors provide only weak constraints on the composition. The investigation of surface composition diversity is helpful to understand the different evolution and the different physical processes affecting the surfaces.

Spectroscopy is the ideal method to investigate the surface composition of these primitive objects. However given their faintness, even if observed with the world's largest telescopes, the visible and near-infrared spectra are available only for a relative small number of them, and in general with very low $\mathrm{S} / \mathrm{N}$. To investigate the surface properties of these remote objects a Large Programme was carried out at VLT-ESO (Cerro Paranal, Chile) to observe them in visible and near- infrared photometry and spectroscopy between 2001 and 2003, together with other single complementary dedicated programmes.

In this paper we present new visible and near-infrared observations of 55638 (2002 $\mathrm{VE}_{95}$ ) obtained at VLT (ESO). A surface model has been derived for this object with two different methods. A comparison with all spectroscopic observations performed by our group at VLT as well as with all the data available in the literature is presented and discussed.

\section{Observations and data reduction}

The observations of the TNO 55638 presented here have been carried out at VLT (Very Large Telescope) at ESO (Chile). Near-infrared photometry and spectroscopy were carried out on October 16th, 2003 with ISAAC 
Table 1. Observational circumstances for 55638 (2002 VE 95$)$ : instrument, heliocentric distance $(r)$, geocentric distance $(\delta)$ in AU and phase angle $(\alpha)$ in degrees.

\begin{tabular}{lcccc}
\hline \hline Date & Instrument & $\begin{array}{c}r \\
(\mathrm{AU})\end{array}$ & $\begin{array}{c}\delta \\
(\mathrm{AU})\end{array}$ & $\begin{array}{c}\alpha \\
(\mathrm{deg})\end{array}$ \\
\hline 16/Oct./2003 & ISAAC & 28.01 & 27.15 & 1.06 \\
\hline $23 /$ Oct./2004 & FORS1 & 28.04 & 27.15 & 0.92 \\
\hline
\end{tabular}

Table 2. Photometric results obtained for 55638 .

\begin{tabular}{lccccccc}
\hline \hline$V$ & $\sigma V$ & $J$ & $\sigma J$ & $H$ & $\sigma H$ & $K$ & $\sigma K$ \\
\hline 20.20 & 0.04 & 17.98 & 0.04 & 17.58 & 0.04 & 17.56 & 0.05 \\
\hline
\end{tabular}

(http://www.eso.org/instruments/isaac). We also carried out visible spectroscopy on October 23rd, 2004 with FORS1 (http://www.eso.org/instruments/fors1). Table 1 lists the details of the observational circumstances.

\subsection{Photometry}

The near-infrared observations were carried out under good photometric conditions. Photometric $J, H$ and $K$ measurements (centered at $1.25,1.65$ and $2.16 \mu \mathrm{m}$ ) were made before each spectroscopic measurement. We used the jitter imaging technique, generating a combined image with the jitter routine from the ECLIPSE package (see Romon et al. 2001). The calibration was performed by observing several faint infrared standard stars from Hunt et al. (1998) and Persson et al. (1998). The data reduction was carried out using the MIDAS package, and the data processing method that was followed is described in Romon et al. (2001a). The photometric results are given in Table 2. The photometric values were transformed into reflectivity using solar color indices $V-J=1.08, J-H=0.29$ and $H-K=0.06$ (Hartmann et al. 1982).

\subsection{Spectroscopy}

Visible spectroscopy of the TNO 55638 was performed with a low resolution grism and a $1^{\prime \prime}$ slit (covering the 4000-9000 Angstrom wavelength range with a spectral resolution of about 550). The visible spectrum was reduced using the software package MIDAS with the standard method (Barucci et al. 2000). Near-infrared spectroscopy was carried out using ISAAC in its SW mode (1-2.5 $\mu \mathrm{m}$ spectral range and equipped with a CCD of $1024 \times 1024$ pixels), with a $1^{\prime \prime}$ slit and grating at three different central wavelengths corresponding to the $J$, $H$ and $K$ bands. The spectral resolution in the three bands was about 500 . The observations were done by nodding the object along the slit by $10^{\prime \prime}$ between two positions A and B. The A and $\mathrm{B}$ images were combined using MIDAS following the procedures described by Romon-Martin et al. (2002) and Barucci et al. (2002). Several solar analogs were observed during each night and each spectrum of the target was divided by the solar analog spectrum observed at similar airmass. The initial spectra have been smoothed with a median filter technique (using a box of $15 \AA$ in the spectral direction for each point of the spectra) to increase the $\mathrm{S} / \mathrm{N}$ ratio and the obtained spectra are shown in Fig. 1. The observational details are reported in Table 3.
Table 3. Spectroscopic observations of the object: Universal time at start exposure (UT), Spectroscopic Range (R), Exposure Time (ET in minutes), Airmass and Analog stars.

\begin{tabular}{lccccc}
\hline \hline Date & UT & $R$ & ET & Airmass & Analog \\
\hline $16 / 10 / 2003$ & $4: 50$ & $H$ & 72 & $1.22-1.34$ & $\begin{array}{c}\text { Landolt 93-101 } \\
\text { HD209847 }\end{array}$ \\
\hline $16 / 10 / 2003$ & $6: 12$ & $J$ & 30 & $1.20-1.22$ & Landolt 115-271 \\
\hline $16 / 10 / 2003$ & $6: 57$ & $K$ & 102 & $1.22-1.48$ & $\begin{array}{c}\text { Landolt 93-101 } \\
\text { HD209847 }\end{array}$ \\
\hline $23 / 10 / 2004$ & $7: 42$ & $V$ & 40 & $1.3-1.45$ & HD76151 \\
\hline
\end{tabular}

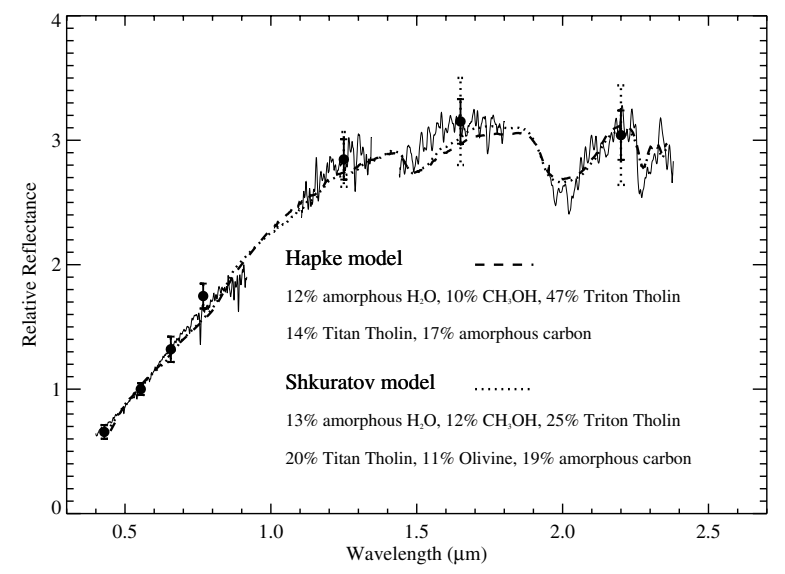

Fig. 1. Spectral reflectances of $556382002 \mathrm{VE}_{95}$ obtained in $V, J, H$ and $K$ ranges (full line) have been adjusted using the photometric $V-J$, $V-H$ and $V-K$ colors and have been normalized to 1 at $0.55 \mu \mathrm{m} . J, H$ and $K$ error bars show the relative errors of the measurements and the errors (vertical dotted lines) that are possible due to the variations of $V$ (amplitude) of the body lightcurve. The obtained intimate models have also been reported.

\section{Observational results and surface models of $55638\left(2002 \mathrm{VE}_{95}\right)$}

55638 (2002 VE95) is a TNO belonging to the Plutino class as defined by its orbit in the 3:2 resonance with Neptune. Ortiz et al. (2005) report photometric variations with an amplitude of $0.08 \pm$ 0.04 but the observations could not distinguish between the two computed periodicities (6.76 or $9.47 \mathrm{~h}$ ).

The visible and near-infrared spectra in the $J, H$ and $K$ bands were not recorded simultaneously and have been combined considering the measured colour indices $V-J=2.22 \pm 0.06$, $J-H=0.40 \pm 0.06$ and $H-K=0.02 \pm 0.06$. As the amplitude of the observed lightcurve is about 0.08 , we report this error (vertical dotted lines) in Fig. 1 as a possible source of error in scaling the individual segments of the spectra. Using the classification method by Barucci et al. (2005b) the object belongs to the taxonomic group RR, which represents the reddest objects of the solar system. The visible spectrum is featureless with a slope between 400 and $850 \mathrm{~nm}$ of $27.9 \pm 0.1 \% / 100 \mathrm{~nm}$. In the near-infrared, the spectrum is featureless in $J$ and shows clear bands in the $K$ region, around $2.0 \mu \mathrm{m}$ and $2.27 \mu \mathrm{m}$. We attributed the $2.0 \mu \mathrm{m}$ band to $\mathrm{H}_{2} \mathrm{O}$. The feature at $2.27 \mu \mathrm{m}$ seems real, even if the last part of the spectrum $(\geq 2.3 \mu \mathrm{m})$ has lower $\mathrm{S} / \mathrm{N}$ and we assign it to the presence of $\mathrm{CH}_{3} \mathrm{OH}$ ice (or a similar molecule). To investigate the possible composition of the surface of this object, we ran two radiative transfer models, based on the Hapke (1981 and 1993) and Shkuratov (1999) theories, considering geographical and intimate mixtures of different materials. We used several minerals, ices and different organic complexes 


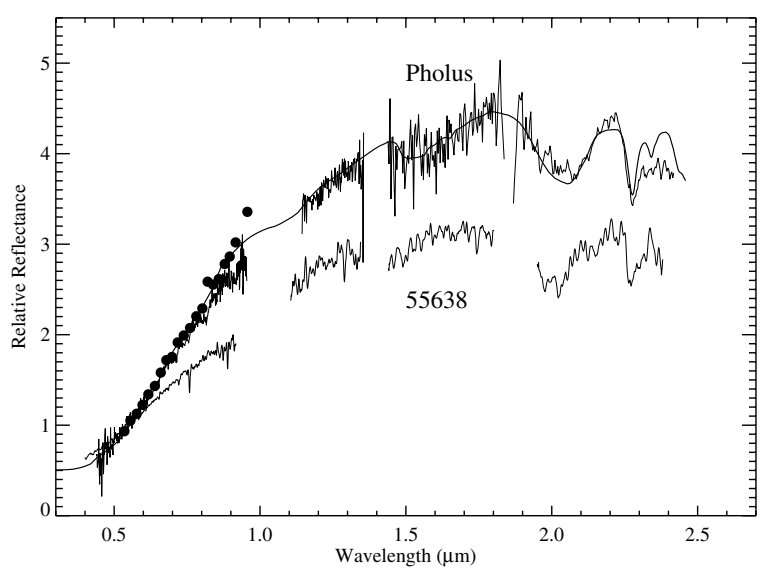

Fig. 2. Relative reflectances of 55638 and of the Centaur Pholus with the computed model (continuous line): Titan tholin, olivine, $\mathrm{H}_{2} \mathrm{O}, \mathrm{CH}_{3} \mathrm{OH}$, and carbon black (Cruikshank et al. 1998).

(e.g. tholins and kerogens) at different grain sizes. The selected model is obtained by best fit using the Levenberg-Marquard minimization algorithm of a mixture in which composition, abundance and grain size are free parameters (Press et al. 1986).

If we assume an albedo of about 0.10 (that is close to the average value for TNOs) at $0.55 \mu \mathrm{m}$, the intimate mixture giving the best match (as measured by $\chi^{2}$ ) over the spectral range between 0.4 and $2.4 \mu \mathrm{m}$ is composed of amorphous $\mathrm{H}_{2} \mathrm{O}$ ice $(30 \mu \mathrm{m}), \mathrm{CH}_{3} \mathrm{OH}$ ice $(40 \mu \mathrm{m})$, Titan tholin and Triton tholin with particles of $4 \mu \mathrm{m}$ size, and amorphous carbon $(10 \mu \mathrm{m})$. With the Shkuratov intimate model, we had to add also some olivine (100 $\mu \mathrm{m}$; Lucey 1998). The amount of each compound depends of the model (see Fig. 1). Tholins are hard, red-brownish substances made of complex organics compounds and they are the best candidates for reproducing the red slope up to $1.2 \mu \mathrm{m}$ (Khare et al. 1984, 1993).

The presented models give only a possible indication of the surface composition as too many unknown parameters influence the computation. The behavior of the spectrum of 55638 is very similar to that of Pholus reported in Fig. 2 (Cruikshank et al. 1998). Pholus is a Centaur which is very red and shows clear evidence of water ice bands and solid methanol (and/or a photolytic product of methanol having small molecular weight). The presence of methanol on the surface of this TNO and the Centaur Pholus would indicate the chemically primitive nature of these bodies. Methanol is an abundant component of many active comets and of the interstellar medium (Bockelee-Morvan et al. 2004; Wooden et al. 2004).

\section{Composition analysis}

Spectroscopic observations are the best technique available to investigate the surface compositions, and are particularly useful in the near-infrared spectral region where signatures of ices are present. From the analysis of the previously observed TNO spectra, the majority of the objects showed spectra with low $\mathrm{S} / \mathrm{N}$ with faint or no signatures (Dotto et al. 2003b; Barucci et al. 2004). Recently, several large objects with strong ice signatures in their spectra have been discovered.

To investigate the presence of ices and their connection with the evolution of the population, we analyze all the NIR spectra available in the literature for which a surface compositional model was performed. Table 4 lists all the objects for which nearinfrared spectra (total sample $=32$ ) have been reported. Pluto

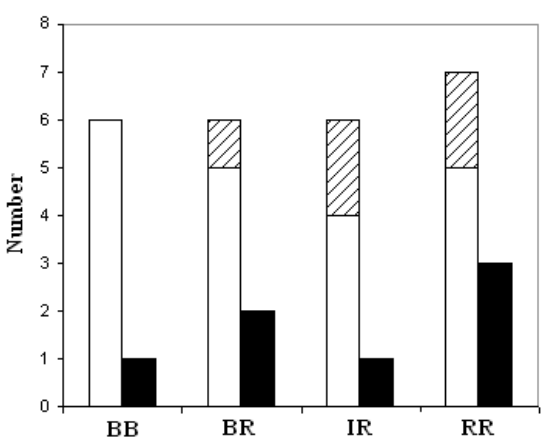

Fig. 3. Distribution of icy (white) and non-icy (black) bodies as a function of their taxonomy. To plot this figure, we used the objects indexed in Table 4. Dashed lines are reported for objects with published models including ices but with no firm detection.

and Charon have also been included in the table, as these objects can be considered members of the same population. We select all the TNOs and Centaurs for which ices seem to be present on their surface from published modelling even if only with a very small percentage $(\geq 2 \%)$. We analyze the presence of ice, as reported by the different authors, and the possible relation with the size of the objects, their surface characteristics (taxonomic groups) and their dynamical properties.

For the surface characteristics and spectral redness we use the taxonomic groups defined by Barucci et al. (2005b) who defined four groups named: BB, BR, IR and RR. The group BB contains objects with neutral color and RR those with very red color, while the others have intermediate behavior. In Fig. 3 we represent the distribution in the four taxonomic groups with ice (white) and no ice (black) present on their surfaces as revealed by their spectra and the computed compositional models. The objects with models including ices but with no firm detection are presented with dashed lines. All four groups show the presence of ices. The majority of non-icy bodies seems concentrated in the RR group where organic compounds (causing red slopes) could cover and hide the majority of ices present on the surface. The BB group, characterized by neutral spectra sometimes bluish in the near-infrared, has objects for which the content of ice seems generally higher than in the other groups, although the presence of $\mathrm{H}_{2} \mathrm{O}$ ice in the Chiron spectrum seems connected to temporal/orbital variations. Only one object in the BB group (1996 TL66) has a featureless spectrum, though the spectrum (Luu \& Jewitt 1998) has very poor S/N.

We also analyzed the connection between the presence of ice and the dynamical properties of the objects. To search for a relationship between the presence of ice and the dynamical classes, we considered separately the classical objects, the resonant objects, and the scattered objects. Classical ones have also been separated in high $\left(\geq 5^{\circ}\right)$ and low $\left(<5^{\circ}\right)$ orbital inclination bodies to investigate differences between the "hot" and "cold" population. No obvious trends with these dynamical classes (see Table 4), or with the perihelion distance (Fig. 4) have been identified.

We also analyzed the connection between the presence of ice and the sizes of the objects. The determination of the diameter is not very precise as shown in Table 4 , where the error is reported for each measurement. When several measurements were available we favored the most accurate values or those obtained by SPITZER observations. In Fig. 4 we report the apparent magnitude in the $H$-band ( $\mathrm{mH}$, measured during the relative observations close to the opposition) versus the perihelion distance for 


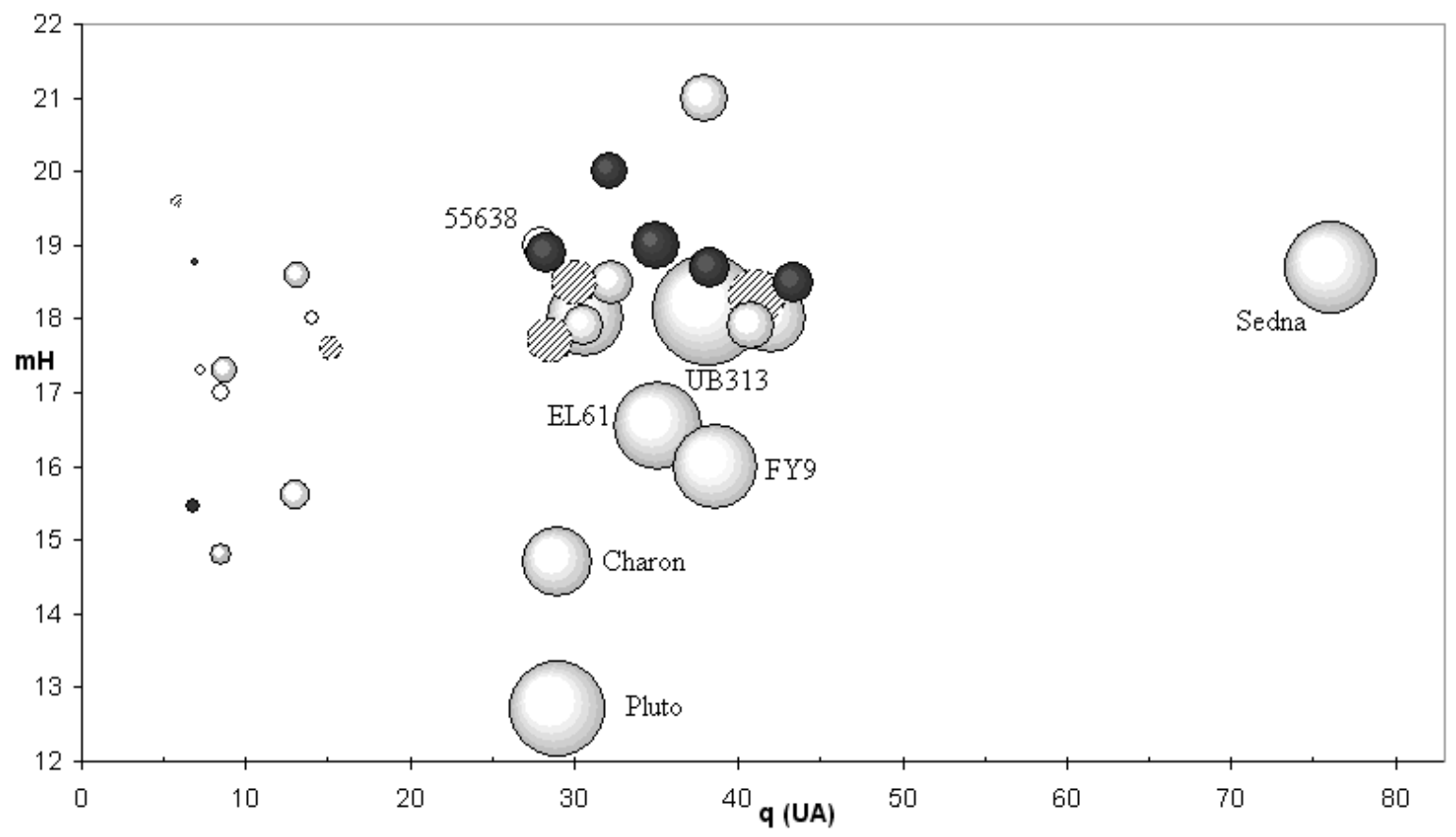

Fig. 4. Distribution of the icy (white) and non-icy (black) bodies superposed to the apparent $H$-band magnitude (mH) vs. perihelion distance ( $q$ ) plot. The objects represented with dashed lines are those whose models include ices but with uncertain data. In the figure, all the Centaurs and the TNOs listed in Table 4 have been represented. Each object has a different symbol size proportional to the diameter dimension according to the values given in Table 4.

all the objects listed in Table 4. The symbol sizes are proportional to the diameters. We used the apparent magnitude $(\mathrm{mH})$ to analyze the bias effect connected with the faintness of the individual objects. From the analysis of the spectra, we notice that spectra of faint objects with no ice detection have in many cases the same quality as those with ice detection and therefore we can assume that our analysis is not affected by a bias due to the faintness of the observed bodies.

The clear result of the analysis, even if performed on a small sample of 32 objects, shows that all large objects have ices detected on their surface, while smaller objects can have no ice detection, small ice detection or heterogeneity on their surface.

Up to now the different compositions have been attributed to different collisional evolution states and/or different degrees of surface alteration due to space weathering. From laboratory experiments (Strazzulla 1997; Strazzulla et al. 1998; Brunetto et al. 2006) it is known that bombardment by high-energy radiation of $\mathrm{H}_{2} \mathrm{O}, \mathrm{CH}_{4}, \mathrm{CH}_{3} \mathrm{OH}, \ldots$...ices, produces an "irradiation mantle" which is hydrogen poor and carbon-rich, reddening the spectra and destroying the ices' bands. We can separate in the analysis the Centaurs from the TNOs as, even if they had similar origin, they have had a different evolution. The probability to have comet activity is higher for Centaurs. However, if the space weathering is the cause (agent) of the formation of a crust, which hides the presence of ices on the surface, why does this process appear not to work on the larger objects?

\section{Conclusions}

We have presented new visible and near-infrared data for the Plutino object 55638 (2002 VE 95 ). The obtained spectra show signatures at 1.5, 2.0 and $2.27 \mu \mathrm{m}$ which implies the detection of $\mathrm{H}_{2} \mathrm{O}$ and $\mathrm{CH}_{3} \mathrm{OH}$ (or a similar molecule). The absorption at $2.27 \mu \mathrm{m}$ had previously only been detected on the Centaur Pholus, and attributed to $\mathrm{CH}_{3} \mathrm{OH}$ (and/or a photolytic product of methanol). This detection indicates a chemically primitive surface, since heating and other processes remove the light hydrocarbons in favor of macromolecular carbon. A model of the surface composition of 55638 has been presented using two different radiative transfer models. The similarity found between the spectral behavior of the TNO 55638 and that of the Centaur 5145 Pholus could imply a common origin for the two bodies.

To investigate the surface properties of the whole population of TNOs and Centaurs, we have analyzed the new result together with all the spectroscopic data available from the VLT-ESO programs on TNOs and Centaurs and those available in the literature. The main results of the analysis on the whole sample of 32 objects can be summarized as follows:

- No obvious trends have been found between the presence/detection of ice and the taxonomic groups (BB, BR, IR, $\mathrm{RR}$ ), though the BB group (objects with neutral/bluish spectra) seems to show more ice content on the surface, and the RR group (the reddest objects) seems to favor objects with no ice detection.

- No clear relationship appears between the presence of ice in near-infrared spectra and the dynamical classes and/or perihelion distance, which could have implied a common origin.

- All large objects $(D \geq 700 \mathrm{~km})$ show the presence of some ices on their surface. Even if we have a very small sample, diameters with large errors, and many poor quality spectra, the fact that all the studied large objects show the presence of ice on their surface means that space weathering and collisions/resurfacing are not the only mechanisms that can explain the surface properties and composition. It is clear that the largest TNOs can retain materials of higher albedo (ice or frost), but they can also have an outgassing from the interior.

Crystalline water ice has been detected on the surface of Quaoar (Jewitt \& Luu 2004) implying that the ice has been heated above 100/110 K temperatures. The presence of crystalline water could imply cryovolcanic outgassing. As discussed by Jewitt \& Luu (2004), complex geology as observed on small 
Table 4. Diameter (in kilometers), ices observed, taxonomic group (Barucci et al. 2005b) and dynamical group (C: Centaurs, Cl: Classical, R: Resonant, S: Scattered and ES: Extended scattered disk) of the TNOs and Centaurs available in the literature. References: (Al04): Altenhoff et al. (2004), (Ba00): Barucci et al. (2000), (Ba02): Barucci et al. (2002), (Ba05a): Barucci et al. (2005a), (Bau02): Bauer et al. (2002), (Be06): Bertoldi et al. (2006), (Boe04) : Boehnhardt et al. ( 2004), (Br98): Brown (1998), (Br99): Brown et al. (1999), (Br00a): Brown (2000), (Br00b): Brown et al. (2000), (Br00c): Brown \& Calvin (2000), (Br04): Brown \& Trujillo (2004), (Br05): Brown et al. (2005), (BrK98): Brown \& Koresko (1998), (Bark): Barkume et al. (2006), (Cr98): Cruikshank et al. (1998), (Cr05): Cruikshank et al. (2005), (Da93): Davies et al. (1993), (dB04): de Bergh et al. (2004), (dB05): de Bergh et al. (2005), (Dor03): Doressoundiram et al. (2003), (Dor05): Doressoundiram et al. (2005), (Dot03a): Dotto et al. (2003a), (Dot03c): Dotto et al. (2003c), (Fe02): Fernandez et al. (2002), (Fo04): Fornasier et al. (2004), (Fo99): Foster et al. (1999), (Gr96): Grundy \& Fink (1996), (Gr05): Grundy et al. (2005), (Gu06): Gulbis et al. (2006), (Je01): Jewitt \& Luu (2001), (Je04): Jewitt \& Luu (2004), (Ke00): Kern et al. (2000), (Li01): Licandro et al. (2001), (Li02): Licandro et al. (2002), (Li05): Licandro et al. (2005), (Li06): Licandro et al. (2006b), (Lu98): Luu \& Jewitt (1998), (Lu00): Luu et al. (2000), (Me05): Merlin et al. (2005), (Mi93): Millis et al. (1993), (Ow93): Owen et al. (1993), (Ra): Rabinowitz et al. (2006), (Ro02): Romon-Martin et al. (2002), (Ro03): Romon et al. (2003), (Sh02): Sheppard \& Jewitt (2002), (Si06): Sicardy et al. (2006), (St): Stansberry, personal communication, (Th89): Tholen \& Buie (1989), (Th00): Thomas et al. (2000), (Tr05): Trujillo et al. (2005), $\left({ }^{+}\right)$: Kern et al. (2000) claimed to have detected water ice on one side, but the results have been contradicted by later observations, $\left({ }^{++}\right)$: Trujillo et al. (2005) obtained a largely featureless spectrum, $(*)$ : if we assume an albedo of 0.10 .

\begin{tabular}{|c|c|c|c|c|}
\hline Object & $\overline{\text { Ices (Ref) }}$ & Diameter (Ref) & Tax. groups & Dyn. groups \\
\hline 2060 Chiron (1977 UB) & $\mathrm{H}_{2} \mathrm{O}$, variable (Lu00, Fo99, Ro03) & $150 \pm 10(\mathrm{Fe} 02)$ & $\mathrm{BB}$ & $\mathrm{C}$ \\
\hline 5145 Pholus (1992 AD) & $\mathrm{H}_{2} \mathrm{O}, \mathrm{CH}_{3} \mathrm{OH}(\mathrm{Cr} 98)$ & $190 \pm 20(\mathrm{Da93})$ & $\overline{\mathrm{RR}}$ & $\mathrm{C}$ \\
\hline 8405 Asbolus (1995 GO) & none $\left(\mathrm{Ke} 00^{+}, \mathrm{Ba} 00, \mathrm{BrO0a}, \mathrm{Ro} 02\right)$ & $75 \pm 10(\mathrm{Fe} 02, \mathrm{St})$ & $\mathrm{BR}$ & $\mathrm{C}$ \\
\hline 10199 Chariklo $\left(1997 \mathrm{CU}_{26}\right)$ & $\mathrm{H}_{2} \mathrm{O}(\mathrm{BrK} 98, \mathrm{Br} 98$, Dot03c $)$ & $230 \pm 30(\mathrm{St})$ & $\mathrm{BR}$ & $\mathrm{C}$ \\
\hline 31824 Elatus $\left(1999 \mathrm{UG}_{5}\right)$ & $\mathrm{H}_{2} \mathrm{O}(\mathrm{Bau} 02)$ & $40 *$ & IR? & $\mathrm{C}$ \\
\hline 32532 Thereus $\left(2001 \mathrm{PT}_{13}\right)$ & $\mathrm{H}_{2} \mathrm{O}(\mathrm{Ba} 02, \mathrm{Me} 05, \mathrm{Li} 05)$ & $85 \pm 15(\mathrm{St})$ & $\mathrm{BR}$ & $\mathrm{C}$ \\
\hline 52872 Okyrhoe $\left(1998 \mathrm{SG}_{35}\right)$ & $\mathrm{H}_{2} \mathrm{O} ?($ Dot03a) & $50 \pm 10(\mathrm{St})$ & $\mathrm{BR}$ & $\mathrm{C}$ \\
\hline 54598 Bienor $\left(2000 \mathrm{QC}_{243}\right)$ & $\mathrm{H}_{2} \mathrm{O}$ (Dot03a) & $180 \pm 30(\mathrm{St})$ & $\mathrm{BR}$ & $\mathrm{C}$ \\
\hline 55576 Amycus $\left(2002 \mathrm{~GB}_{10}\right)$ & $\mathrm{H}_{2} \mathrm{O}$ ? (Dor05) & $120 *$ & $\mathrm{RR}$ & $\mathrm{C}$ \\
\hline $63252\left(2001 \mathrm{BL}_{41}\right)$ & none (Dor03) & $20 *$ & $\mathrm{BR}$ & $\mathrm{C}$ \\
\hline 83982 Crantor $\left(2002 \mathrm{GO}_{9}\right)$ & $\mathrm{H}_{2} \mathrm{O}$ (Dor05) & $65 *$ & $\mathrm{RR}$ & $\mathrm{C}$ \\
\hline $15789(1993 \mathrm{SC})$ & none $(\mathrm{Je} 01)$ & $330 \pm 66(\mathrm{Th} 00)$ & $\overline{\mathrm{RR}}$ & $\overline{\mathrm{R}}$ \\
\hline $15874\left(1996 \mathrm{TL}_{66}\right)$ & none (Lu98) & $630 \pm 90($ Th00) & BB & $\mathrm{S}$ \\
\hline $19308\left(1996 \mathrm{T0}_{66}\right)$ & $\mathrm{H}_{2} \mathrm{O}(\mathrm{Br} 99)$ & $<900$ (A104) & BB & $\mathrm{Cl}$ \\
\hline 20000 Varuna $\left(2000 \mathrm{WR}_{106}\right)$ & $\mathrm{H}_{2} \mathrm{O}(\mathrm{Li} 01)$ & $586(-120,+190)(\mathrm{St})$ & IR & $\mathrm{Cl}$ \\
\hline $26181\left(1996 \mathrm{GQ}_{21}\right)$ & none (Dor03) & $400 *$ & $\mathrm{RR}$ & $\mathrm{S}$ \\
\hline $26375\left(1999 \mathrm{DE}_{9}\right)$ & $\mathrm{H}_{2} \mathrm{O}(\mathrm{Je} 01)$ & $500 *$ & IR & $\mathrm{S}$ \\
\hline 28978 Ixion $\left(2001 \mathrm{KX}_{76}\right)$ & $\mathrm{H}_{2} \mathrm{O}$ ? (Li02, Boe04) & $480 \pm 140(\mathrm{St})$ & IR? & $\mathrm{R}$ \\
\hline 38628 Huya $\left(2000 \mathrm{~EB}_{173}\right)$ & $\mathrm{H}_{2} \mathrm{O}$ ? (Br00b, Je01, dB04) & $500 \pm 70(\mathrm{St})$ & IR & $\mathrm{R}$ \\
\hline $47171\left(1999 \mathrm{TC}_{36}\right)$ & $\mathrm{H}_{2} \mathrm{O}(\operatorname{Dot} 03 \mathrm{a}, \mathrm{Me} 05)$ & $420 \pm 65(\mathrm{St})$ & $\mathrm{RR}$ & $\mathrm{R}$ \\
\hline $47932\left(2000 \mathrm{GN}_{171}\right)$ & none $(\mathrm{dB} 04)$ & $400(\mathrm{Sh} 02)$ & IR & $\mathrm{R}$ \\
\hline 50000 Quaoar $\left(2002 \mathrm{LM}_{60}\right)$ & $\mathrm{H}_{2} \mathrm{O}(\mathrm{Je} 04)$ & $1280 \pm 190(\mathrm{Br} 04)$ & RR? & $\mathrm{Cl}$ \\
\hline $55565\left(2002 \mathrm{AW}_{197}\right)$ & $\mathrm{H}_{2} \mathrm{O}$ ? (Dor05) & $700 \pm 50(\mathrm{Cr} 05)$ & IR & $\mathrm{Cl}$ \\
\hline 55638 (2002 VE 95$)$ & $\mathrm{H}_{2} \mathrm{O}, \mathrm{CH}_{3} \mathrm{OH}$ (this work) & $400 *$ & RR & $\mathrm{R}$ \\
\hline $79360\left(1997 \mathrm{CS}_{29}\right)$ & none $(\mathrm{Gr} 05)$ & $400 *$ & RR & $\mathrm{Cl}$ \\
\hline 90377 Sedna $\left(2003 \mathrm{VB}_{12}\right)$ & $\mathrm{CH}_{4}, \mathrm{~N}_{2}\left(\mathrm{Ba} 05 \mathrm{a}, \operatorname{Tr} 05^{++}\right)$ & $2000 *$ & $\mathrm{RR}$ & ES \\
\hline 90482 Orcus (2004 DW) & $\mathrm{H}_{2} \mathrm{O}(\mathrm{Fo} 04, \mathrm{~dB} 05, \operatorname{Tr} 05)$ & $1500 *$ & $\mathrm{BB}$ & $\mathrm{R}$ \\
\hline$\left(2003 \mathrm{UB}_{313}\right)$ & $\mathrm{CH}_{4}(\mathrm{Br} 05)$ & $3000 \pm 300(\mathrm{Be} 06)$ & BB & $\mathrm{S}$ \\
\hline$\left(2003 \mathrm{EL}_{61}\right)$ & $\mathrm{H}_{2} \mathrm{O}$ (Bark) & $2000 \pm 500(\mathrm{Ra})$ & $\mathrm{BB}$ ? & $\mathrm{R}$ \\
\hline$\left(2005 \mathrm{FY}_{9}\right)$ & $\mathrm{CH}_{4}(\mathrm{Li} 06)$ & $1800 \pm 500(\mathrm{Li} 06)$ & $\mathrm{BR}$ & $\mathrm{R}$ \\
\hline Pluto & $\mathrm{CH}_{4}, \mathrm{CO}, \mathrm{N}_{2}(\mathrm{Ow} 93)$ & $2350 \pm 60(\mathrm{Mi} 93$, Th89) & $\overline{B R}$ & \\
\hline Charon & $\mathrm{H}_{2} \mathrm{O}, \mathrm{NH}_{3}(\mathrm{Br} 00 \mathrm{c})$ & $1208 \pm 15(\mathrm{Si06}, \mathrm{Gu} 06)$ & $\mathrm{BB}$ ? & \\
\hline
\end{tabular}

icy satellites indicates that solid-state convection that could bring warm ices toward the surface operates on such objects. The feature at $1.65 \mu \mathrm{m}$ which is characteristic of crystalline water ice has also been detected in spectra of Pluto's satellite Charon (Brown \& Calvin 2000). Several objects like Orcus (de Bergh et al. 2005), and 2002 TX300 (Licandro et al. 2006a), even if they have a relatively poor $\mathrm{S} / \mathrm{N}$, have spectra that favour the presence of crystalline water ice. The quality of most of other spectra is too poor to distinguish between amorphous or crystalline water ice or the presence of small features like that of $\mathrm{N}_{2}$ at $2.15 \mu \mathrm{m}$ (except for Sedna).

Abundant methane ice has been found on large TNOs (Licandro et al. 2006bb; Brown et al. 2005), which suggests the need for an interior source to replenish the methane. The presence of methane ice on objects such as 2003 UB313, 2005 FY9, and Sedna (Barucci et al. 2005a), as well as on Pluto, but not on smaller objects, suggests that methane is retained on only the big objects where escape rates are low (Trafton et al. 1997). A thin atmosphere could be formed around some of these objects, as is the case for Pluto, with sublimation and recondensation of the most volatile species depending on the season that would lead to surface modifications.

Even if this field benefits from continuous new discoveries, the investigation of the surface properties is still relatively recent. To investigate the surface of this faint population, albedo remains one of the fundamental parameters to be determined with accuracy. Observations of higher spectral quality and a much more extended sample of objects are also obviously required.

Acknowledgements. We thank E. Quirico for providing us with optical constants of methanol and J. Emery for his helpful review of the manuscript. 


\section{References}

Altenhoff, W. J., Bertoldi, F., \& Menten, K. M. 2004, A\&A, 415, 77 Barkume, K. M., Brown, M. E., \& Schaller, E. L. 2006, ApJ, 640, L87 Barucci, M. A., \& Peixinho, N. 2006, Asteroids, Comets, and Meteors, ed. D. Lazzaro, S. Ferraz-Mello, \& J.A. Fernandez, IAU Symp. 229, 171 Barucci, M. A., de Bergh, C., Cuby, J. G. et al. 2000, A\&A, 357, 53 Barucci, M. A., Boehnhardt, H., Dotto, E., et al. 2002, A\&A, 392, 335 Barucci, M. A., Doressoundiram, A., \& Cruikshank, D. P. 2004, Comets II, ed. M.C Festou, H.U. Keller \& H.A. Weaver (Univ. of Arizona Press), 647 Barucci, M. A., Cruikshank, D. P., Dotto, E., et al. 2005a, A\&A, 439, L1 Barucci, M. A., Belskaya, I. N., Fulchignoni, M., \& Birlan, M. 2005b, AJ, 130, 1291

Bauer, J. M., Meech, K. J., Fernandez Y. R. et al. 2002, PASP, 114, 1309 Bertoldi, F., Altenhoff, W., Weiss, A., et al. 2006, Nature, 439, 563

Bockelee-Morvan, D., Crovisier, J., Mumma M. J., \& Weaver, H. A. 2004

Comets II, ed. M. C. Festou, H. U. Keller, \& H. A. Weaver (Univ. of Arizona Press), 391

Boenhnhardt, Bagnulo, S., Muinonen, K., et al. 2004 A\&A, 415, L21

Brown, M. E. 1998, ApJ, 505, L65

Brown, M. E. 2000, AJ, 119, 977

Brown, M. E., \& Calvin, W. M. 2000, Nature, 287, 107

Brown, M. E., \& Koresko, C. C. 1998, ApJ, 505, L65

Brown, M. E., \& Trujillo, C. 2004, ApJ, 127, 2413

Brown, R. H., Cruikshank, D. P., \& Pendleton, Y. 1999, AJ, 519, 101

Brown, M. E., Blake, G. A., \& Kessler, J. E. 2000, ApJ, 543, L163

Brown, M. E., Trujillo, C., \& Rabinowitz, D. L. 2005, AJ, 635, L97

Brunetto, R., Barucci, M. A., Dotto, E., \& Strazulla, G. 2006, ApJ, 644, 646

Cruikshank, D. P., Roush, T. L., Bartholomew, M. J., et al. 1998, Icarus, 135, 389

Cruikshank, D. P., Stansberry, J. A., Emery, J. P., et al. 2005, ApJ, 624, 53

Davies, J. K., Sykes, M. V., \& Cruikshank, D. P. 1993, Icarus, 102, 166

de Bergh, C., Boehnhardt, H., Barucci, M. A., et al. 2004, A\&A, 416, 791

de Bergh, C., Delsanti, A., Tozzi, G. P., et al. 2005, A\&A, 437, 1115

Doressoundiram, A., Tozzi, G. P, Barucci, M. A., et al. 2003, AJ, 125, 2721

Doressoundiram, A., Barucci, M. A., Tozzi, G.P., et al. 2005, Plan. Space Sci., 53,1501

Dotto, E., Barucci, M. A., Boehnhardt, H., et al. 2003a, Icarus, 162, 408

Dotto, E., Barucci, M. A., \& de Bergh, C. 2003b, CR Acad. Sci., ed. M. A Barucci, Physique tome 4, No. 7, 775

Dotto, E., Barucci, M. A., Leyrat, C., et al. 2003c, Icarus, 164, 122

Duncan, M. J., Levison, H. F., \& Budd, S. M. 1995, AJ, 110, 3073

Fernandez, Y. R., Jewitt, D. C., \& Sheppard, S. S. 2002, AJ, 123, 1050

Fornasier, S., Dotto, E., Barucci, M. A., \& Barbieri, C. 2004, A\&A, 422, L43

Foster, M. J., Green, S. F., McBride, N., \& Davies, J. K. 1999, Icarus, 141, 408 Grundy, W. M., \& Fink, U. 1996, Icarus, 124, 329

Grundy, W. M., Buie, M. W., \& Spencer, J. R. 2005, AJ, 130, 1299

Gulbis, A. A. S., Elliot, J. L., Person, M. J., et al. 2006, Nature, 439, 48
Hapke, B. 1981, J. Geo. Res., 86, 3039, 3055

Hapke, B. 1993, Topics in Remote Sensing (Cambridge, MA: Cambridge University Press)

Hartmann, W. K., Cruikshank, D. P., \& Degewij, J. 1982, Icarus, 52, 377

Hunt, L. K., Mannucci, F., Testi, L., et al. 1998, AJ, 115, 2594

Jewitt, D., \& Luu, J. 1993, Nature, 362, 730

Jewitt, D., \& Luu, J. 2001, AJ, 122, 2099

Jewitt, D., \& Luu, J. 2004, Nature, 432, 731

Kern, S. D., McCarthy, D. W., Buie, M. W., et al. 2000, AJ, 542, L155

Khare, B. N., Sagan, C., Arakawa, E. T., et al. 1984, Icarus, 60, 127

Khare, B. N., Thompson, W. R., Cheng, L., et al. 1993, Icarus, 103, 290

Licandro, J., \& Pinilla-Alonso, N. 2005, ApJ, 630, L93

Licandro, J., Oliva, E., \& Di Martino, M. 2001, A\&A, 373, L29

Licandro, J., Ghinassi, F., \& Testi, L. 2002, A\&A, 388, L9

Licandro, J., di Fabrizio, L., Pinilla-Alonso, N., et al. 2006a, A\&A, in press

Licandro, J., Pinilla-Alonso, N., Pedani, M. et al. 2006b, A\&A, 445, L35

Lucey, P. G. 1998, J. Geoph. Res., 103, 170

Luu, J., \& Jewitt, D. 1998, ApJ, 494, L117

Luu, J., Jewitt, D., \& Trujillo, C. 2000, ApJ, 531, L151

Merlin, F., Barucci, M. A., Dotto, E., et al. 2005, A\&A, 444, 977

Millis, R. L., Wasserman, L. H., Franz, O. G., et al. 1993, Icarus, 105, 282

Ortiz, J. L., Gutierrez, P. J., Santos-Sanz, P., et al. 2005, IAU Symp., 229, abstract ACM P6.15

Owen, T. C., Roush, T. L., Cruikshnak, D. P., et al. 1993, Science, 261, 745

Persson, S. E., Murphy, D. C., Krzeminski, W., et al. 1998, AJ, 116, 2475

Press, W. H., Flannery, B. P., Teukolsky, S. A., \& Vetterling, W. T. 1986, Numerical Recipes (Cambridge University Press)

Rabinowitz, D. L., Barkume, K., Brown, M. E., et al. 2006, ApJ, 639, 1238

Romon, J., de Bergh, C., Barucci, M.A., et al. 2001, A\&A, 376, 310

Romon, J., Delahodde, C., Barucci, M. A., et al. 2003, A\&A, 400, 369

Romon-Martin, J., Barucci, M. A., de Bergh, C., et al. 2002, Icarus, 160, 59

Shkuratov, Y., Starukhina, L., Hoffmann, H., \& Arnold, G. 1999, Icarus, 137, 235

Sheppard, S. S., \& Jewitt, D. C. 2002, AJ, 124, 1757

Sicardy, B., Bellucci, A., Gendro, E., et al. 2006, Nature, 439, 52

Strazzulla, G. 1997, Adv. Space Res., 19, 1077

Strazzulla, G., in Schmitt, B., de Bergh, C., \& Festou, M. 1998, Solar System Ices, Ast. Space Sci. Library, 281 (Dordrecht: Kluwer Academic)

Tholen, D. J., Buie, M. W. 1989, BAAS., 21, 981

Thomas, N., Eggers, S., Lichtenberg, G., et al. 2000, J. Geo. Res., 92, 14933

Trafton, L. M., Hunten, D. M., Zahnle, K. J., \& McNutt, R. L Jr. 1997, Pluto and Charon, ed. S. A. Stern, \& D. J. Tholen (Univ. of Arizona Press), 475

Trujillo, C. A., Brown, M. E., Rabinowitz, D. L., \& Geballe, T. R. 2005, ApJ, 627, 1057

Wooden, D. H., Charnley, S. B., \& Ehrenfreud, P. 2004. Comets II, ed. M. C. Festou, H. U. Keller, \& H. A. Weaver (Univ. of Arizona Press), 33 